\title{
TTR
}

Traduction, terminologie, re?daction

\section{L'emploi des déterminants avec les noms de parenté dans la Métamorphose de Kafka : un problème de traduction}

\section{Joseph Pattee}

Volume 5, numéro 2, 2e semestre 1992

Kafka pluriel : réécriture et traduction

URI : https://id.erudit.org/iderudit/037126ar

DOI : https://doi.org/10.7202/037126ar

Aller au sommaire du numéro

Éditeur(s)

Association canadienne de traductologie

ISSN

0835-8443 (imprimé)

1708-2188 (numérique)

Découvrir la revue

Citer cet article

Pattee, J. (1992). L’emploi des déterminants avec les noms de parenté dans la Métamorphose de Kafka : un problème de traduction. TTR, 5(2), 141-167.

https://doi.org/10.7202/037126ar

Tous droits réservés (C) TTR: traduction, terminologie, rédaction — Les auteurs, 1992

Ce document est protégé par la loi sur le droit d'auteur. L'utilisation des services d'Érudit (y compris la reproduction) est assujettie à sa politique d'utilisation que vous pouvez consulter en ligne.

https://apropos.erudit.org/fr/usagers/politique-dutilisation/ 


\section{L'emploi des déterminants avec les noms de parenté dans la Métamor- phose de Kafka: un problème de traduction $^{1}$}

\section{Joseph Pattee}

$\mathrm{M}^{\mathrm{m} \varepsilon}$ B. Vergne-Cain et M. G. Rudent, traducteurs de la Métamorphose publiée dans la collection bilingue du Livre de Poche, attirent l'attention, dans leur préface ${ }^{2}$, sur le fait que le possessif y est peu utilisé. Leur affirmation semble se rapporter à l'emploi de ce déterminant avec les noms d'objets, les noms de parties du corps aussi bien que les noms de parenté. Mais les quelques commentaires qu'ils font au fil du texte (pp. 28 et 68) concernent uniquement la désignation des membres de la

1. Pour le lecteur peu familiarisé avec la nouvelle de Kafka, voici le résumé qu'en font H.A. et E. Frenzel dans Daten deutscher Dichtung: Le voyageur de commerce Gregor Samsa se réveille un matin transformé en un gigantesque insecte. $\mathrm{Ni}$ lui, ni sa famille [son père, sa mère et sa scur] ne savent que faire de sa personne, maintenant qu'il ne peut plus assumer sa fonction d'unique pourvoyeur de la famille. Dans sa chambre-prison, on montre de moins en moins de bonne volonté à s'occuper de lui, jusqu'à ce que le vou de la famille soit exaucé et qu'il la délivre de sa présence en mourant de faim. Une fois que la servante s'est débarrassée du cadavre, la famille respire à nouveau.

2. Tous nos exemples sont tirés de la traduction de la Métamorphose parue dans le Livre de Poche, à moins qu'il ne soit fait mention expressément des trois autres traductions; elles apparaissent toutes les quatre dans la bibliographie. 
famille. C'est la raison pour laquelle nous nous en sommes tenu à ce type de mots.

Les traducteurs notent qu'avec ces termes Kafka utilise surtout l'article défini ${ }^{3}$, alors que le possessif aurait convenu tout aussi bien; ils décrivent l'effet, un effet de distanciation, obtenu grâce à l'emploi de l'article, mais n'avancent pas de raison pour justifier celui-ci. Pour notre propos - qui porte sur un problème de traduction -, il est intéressant de voir qu'à la suite de cette observation, les deux traducteurs ont pris le parti, pour respecter le texte original, de traduire, dans l'ensemble de l'œuvre, l'article du texte original allemand par l'article français. Ont-ils eu raison? Peut-on transposer tel quel un déterminant par fidélité au texte de départ? Ne risque-t-on pas alors de trahir l'esprit de la langue d'arrivée? Ces questions méritent d'être posées lorsque l'on voit que les trois autres traducteurs de la même œuvre se sont crus obligés, à des degrés divers, de recourir très souvent au possessif en français là où la version originale présentait l'article.

Pour apporter une réponse aux questions que nous venons de poser, il faut d'abord dire ce qui sépare les deux déterminants, l'article défini et le possessif, puis montrer que leur champ de compétence differe en allemand et en français, faire voir comment Kafka lui-même a réparti les déterminants dans sa nouvelle et comment les traducteurs à leur tour les ont distribués. Ce n'est qu'alors que nous serons à même de fournir des éléments de réponse.

\section{L'article défini}

Un des emplois fréquents de l'article est l'emploi anaphorique, qui consiste à rappeler une réalité déjà introduite dans le discours sous la forme d'un substantif précédé de l'article indéfini'. Par exemple:

(1) Der König legte [das Kind] in eine Schachtel und ritt damit weiter, bis er zu einem tiefen Wasser kam: da warf er die Schachtel hinein. (Gr 27)

3. Dans toute notre étude, lorsqu'il sera question d'article, ce sera toujours, sauf indication expresse, de l'article défini.

4. Ou de l'article $d u$, de la, des (de l'article zéro en allemand). 
(Le roi plaça l'enfant dans une boîte et continua sa chevauchée jusqu'à ce qu'il parvienne à un cours d'eau profond. Alors il y jeta la boîte.)

Ce n'est pas là, cependant, le point de départ le plus commode pour illustrer la spécificité de l'article défini par rapport au possessif. Son emploi dans l'anaphore dite associative est plus approprié. L'anaphore associative consiste, le plus souvent, à rappeler au moyen de l'article non pas la même réalité comme en (1), mais une réalité impliquée en tant que partie dans une réalité déjà évoquée dans le discours au titre du tout. On peut illustrer l'anaphore associative par l'exemple suivant, où les deux substantifs (Mauer et Fenster) désignent des parties du tout que représente l'édifice désigné par le premier (Schule), précédemment évoqué:

(2) Vor der Schule stand natürlich ein lebender Zaun, ja, es stimmte, es fehlte nichts. [...] Nicht einmal die Halbkreise draußen an den Mauern unter den Fenstern. (Ha 88)

(Devant l'école, il y avait naturellement une haie vive. [...] Dehors, pas même les demi-cercles sur les murs sous les fenêtres.)

Dans cet exemple, tous les objets désignés au moyen de l'article et du substantif (sans complémentation) constituent des objets que l'on associe d'emblée à l'évocation du lieu qui les renferme. Une des conditions qui régit l'anaphore associative est celle-ci: il faut que le rapport qui unit les désignés (Schule à Mauer et Fenster) ne se limite pas, dans l'expérience, à eux seuls ("cette école a des fenêtres et des murs»), mais s'étende à plusieurs désignés de la même espèce («toute école a des fenêtres et des murs»); il faut, de plus, que le fait fasse partie de l'expérience d'une collectivité.

Toutefois, on observe parfois, dans un autre type de contexte, que l'on peut utiliser l'article défini, lorsque plusieurs substantifs sont en cause, si les êtres ou les objets dont on parle forment un ensemble uniquement dans l'expérience d'une ou de quelques personnes. Il faut néanmoins que tous ces êtres, dans le cadre de l'expérience de cette personne, forment un ensemble - ce qui implique que leur réunion n'est pas fortuite ou passagère; il faut donc qu'ils aient été réunis à 
plusieurs reprises, les activités dans lesquelles ils sont engagés acquérant par là le statut d'habitude. Le passage suivant de W. Koeppen est caractéristique à cet égard. Le narrateur raconte:

(3) Die Anrede zauberte das Bild schöner Tage. Aufrecht schritt der Musikmeister an der Spitze des Regiments durch die Stadt. Aus Felle und Blech dröhnte der Marsch. Schellen rasselten. Die Fahne hoch. Die Platzmusik im Waldpavillon! Der Meister dirigierte den Freischütz. (Koe 16)

(Le discours lui fit revoir comme par magie un tableau des vieux jours: le chef d'orchestre s'avançait bien droit à la tête du régiment à travers la ville, les tambours jouaient la marche retentissante. On faisait sonner les clochettes. Haut le drapeau. La musique dans le kiosque du bois. Le chef dirigeait le «Freischütz».)

Ainsi, lorsque plusieurs réalités sont en cause, l'article est lié à l'impression des parties d'un tout, leur rapport étant jugé comme un fait largement répandu (anaphore associative) ou, au contraire, limité à un seul ensemble, mais pour une période de temps relativement étendue la jeunesse du narrateur dans l'exemple (3). En d'autres termes, dans les deux cas, l'impression liée à l'emploi de l'article est celle de plusieurs réalités qui forment un ensemble dont l'existence a acquis une certaine permanence, parce que ces réalités forment fréquemment un ensemble dans l'expérience d'une collectivité ou d'un individu.

Dans le cas qui nous occupe, les noms de parenté dans la Métamorphose, l'effet de sens lié à l'emploi de l'article défini est bien celui que nous venons de décrire. Der Vater/die Mutter/die Schwester (le père, la mère et la sœur), en effet, constituent trois éléments englobés dans un ensemble, la famille. Les deux premiers comme éléments communs à toute famille et le dernier comme élément que l'on trouve dans plusieurs familles et dans celle-ci en particulier. On observe que l'article, grammaticalement, suggère l'image de parties d'un ensemble, mais ces parties ne sont pas liées expressément à Gregor. On pourrait illustrer de la façon suivante l'image que suscite l'emploi de l'article, le grand rectangle symbolisant la famille: 


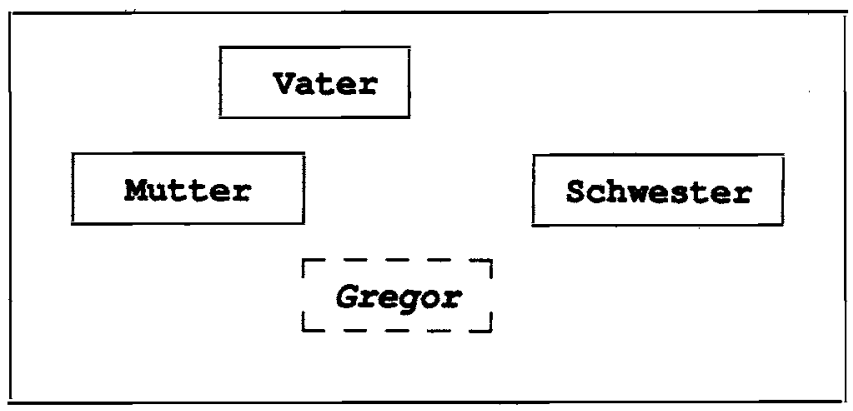

Il existe bien un lien entre les personnes désignées par les noms de parenté et Gregor, mais il est le fait du substantif lui-même; les noms de parenté étant, sémantiquement, des termes relationnels.

\section{Le possessif ${ }^{5}$}

Il est banal aujourd'hui de signaler que le possessif n'indique pas toujours la «possession». C'est pourquoi nous dirons plutôt qu'il signifie la référence à un être en liaison avec son rang personnel, qui peut être premier, second ou troisième, selon le rôle qu'il joue dans le rapport interlocutif. Cette définition, volontairement extensive, s'accorde mieux, en effet, à l'ensemble des emplois du possessif.

Que peut-on exprimer à l'aide du possessif avec des substantifs autres que les noms de parenté? Prenons, par exemple:

(4) Ein ausgetüfteltes System von elektrischen Lampen sorgte dafür, daß Jonathan an drei verschiedene Stellen seines Zimmers - nämlich am Fuß-und am Kopfende seines Bettes sowie an seinem Tischchen - sitzen und lesen konnte, ohne

5. Nous considérons un syntagme avec complément du nom comme Jonathans Bett ou das Bett des Patienten comme une variante du possessif sein Bett, mais nous n'envisageons ici que l'opposition entre das Bett et sein Bett, ou en fait, entre der Vater et sein Vater. 
geblendet $\mathrm{zu}$ werden und ohne daß ein Schatten auf die Zeitung fiel. (Sü 11)

(Une ingénieuse disposition des lampes électriques faisait qu'en trois différents endroits de sa chambre - à savoir au pied comme à la tête de son lit, et devant sa petite table Jonathan pouvait s'asseoir et lire son journal sans être ébloui et sans faire de l'ombre.)

Le possessif sein contient une troisième personne, comme le nom Jonathan. Du fait que cette troisième personne présente dans le possessif et dans le nom propre désigne le même individu, il est alors établi un rapport entre le possesseur et le possédé. L'article, ici, aurait suggéré deux objets faisant d'ordinaire partie, l'un et l'autre, d'une chambre à coucher, indépendamment de son occupant, comme dans ce passage qui fait suite immédiatement au précédent:

Am Kopfende des Bettes hatte er ein Regal angebracht, in dem nicht weniger als siebzehn Bücher standen. (Sü 11)

(À la tête du lit, il avait installé une étagère, où il n'y avait pas moins de dix-sept livres.)

Quel sera l'effet de l'emploi du possessif avec les noms de parenté? Dans un syntagme tel que sein Vater, seine Mutter, seine Schwester, le rapport à Gregor sera double: l'on aura, lexicalement, toujours l'expression d'un rapport à Gregor avec le substantif et, surimposé, pour ainsi dire, grammaticalement, l'expression d'un rapport à Gregor avec le déterminant sein. On pourrait illustrer la relation qu'institue le possessif de la façon suivante:

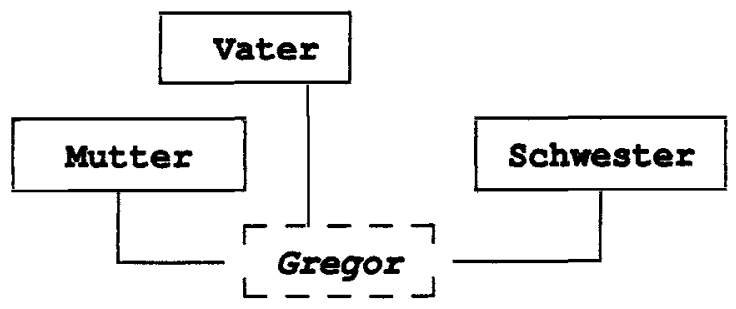


De plus, un effet régulier du possessif est de donner l'impression que chaque réalité est indépendante des autres, parce que ces réalités ne font pas partie d'un ensemble qui les subsume.

Il importe de bien distinguer deux ordres de réalité: les faits grammaticaux qui représentent la façon dont le sujet parlant/écrivant saisit et voit la réalité et les faits mêmes de la réalité. Nous connaissons la situation dans la famille de Gregor, le lien qui unit les quatre personnages. Cependant, quand l'auteur désigne les membres de la famille au moyen de l'article, il oblitère, au profit d'un ensemble - la famille - le lien qui les unit à Gregor, lien qui demeure alors uniquement lexical. Au contraire, avec l'emploi du possessif, l'accent est mis davantage sur le lien qui les unit à Gregor, en oblitérant le fait qu'ils forment à eux tous une cellule familiale.

\section{La répartition de l'article défini et du possessif}

On observe de façon très générale que l'allemand, contrairement au français, fait un plus large usage de l'article que du possessif dans les contextes où les deux déterminants peuvent entrer en concurrence.

1. Dans les deux langues, on emploie l'article lorsqu'il s'agit d'une partie du corps, que celle-ci est l'objet d'un verbe de mouvement et qu'il s'agit d'une partie du corps du sujet. Par exemple:

(6) Ich war zu müde, um den Kopf zu heben. (Segh 8) (J'étais trop fatigué pour relever la tête.)

2. Avec d'autres verbes, qui autorisent plus facilement le possessif, l'emploi de ce dernier avec le nom des parties du corps (ou des facultés de l'âme) a régulièrement cet effet de «détacher» la partie de la personne, de la rendre autonome, tandis que l'article, au contraire, va souligner sa qualité de partie du corps. Hatcher écrit à ce propos: «The adjectives mon, ton... represent [the part of the body] as an object detached from his personal sphere»; "with the article, however, the part is represented as an integral part of the person» (Hatcher, 1944, p. 457). L'opposition qu'elle caractérise par les termes de «solidarity» et «detachment» donne lieu à toutes sortes d'effets de sens, avec des substantifs désignant autre chose que des parties du corps à proprement 
parler et avec d'autres verbes que des verbes de mouvement. L'exemple (7) est intéressant de ce point de vue. On peut lire à deux lignes de distance:

(7) Er wünschte, daß er sein Bewußtsein verlieren ... möge. Er strenge sich mit aller Macht an, das Bewußtsein zu verlieren. (Sü 82)

(Il souhaitait perdre connaissance ... Il cherchait de toutes ses forces à perdre connaissance.)

Dans le contexte antérieur, il est rapporté que le personnage pouvait, dans son enfance, s'évanouir à volonté. Or, voilà qu'au moment où il veut à tout prix perdre connaissance (sein Bewußtsein verlieren), cela ne lui est plus possible. La perte de conscience n'est plus quelque chose qu'il peut contrôler et provoquer de lui-même (das Bewußtsein verlieren).

On voit que, quand l'alternance est possible, le possessif souligne l'autonomie du désigné et, par là, souligne également le contrôle conscient que la personne peut exercer sur lui parce qu'il est vu extérieur à lui, manipulable pour ainsi dire; ce qui ne serait pas possible, si le désigné, avec l'article, était présenté comme une partie intégrante de la personne ${ }^{6}$.

6. Pour des précisions, le lecteur se reportera à l'article de $\mathrm{M}^{\mathrm{mo}}$ Hatcher (1944, p. 476) où elle explique tous les jeux stylistiques auxquels se prête l'opposition des deux déterminants. Nous ne citerons que sa conclusion : «In the various contrasts analysed above we have found always the same motifs recurring : the typical vs. the original; the abstract vs the concrete; pure movement, self determined, vs. activity directed toward a terminus; natural vs. constrained movement; the static vs. the dynamic. These distinctions are possible because the article, ... is itself representative of the 'normal' : the ideal, the natural (the easy), the typical. To this the Poss. with its emphasis on the individualized part, must of necessity introduce the contrasting idea of non-conformity.» 
Jusqu'ici, l'allemand et le français ne different pas sensiblement. On note, cependant, que, dans d'autres conditions, l'allemand présente plusieurs différences par rapport au français.

3. L'une de ces conditions se présente lorsqu'il y a un apport sémantique au substantif. G. Guillaume note (Guillaume, 1919, p. 214) que, par exemple, l'adjonction d'une qualité de caractère momentanée affectant la partie du corps exige, en français, de renoncer à l'article et de recourir au possessif (Il tendit sa main rougie). C'est aussi souvent le cas en allemand. On dira, sans apport sémantique, avec l'article:

(8) Sie schob die Brille mit den Fingern ein wenig die Nase hinauf, um Jonathan besser ins Auge fassen zu können. (Sü 68) (Littéralement: Elle repoussa un peu les lunettes du bout des doigts ...)

(Elle repoussa un peu ses lunettes du bout des doigts vers le haut de son nez pour mieux considérer Jonathan.)

puis suivi d'un apport sémantique, avec le possessif:

(9) «Ja, natürlich, sagte Madame Topell und schob ihre Brille, die während der Untersuchung des Loches etwas herabgerutscht war, wieder die Nase hinauf.» (Sü 71)

(«Oui, naturellement», dit $M^{\text {me }}$ Topell en remontant sur son nez ses lunettes qui avaient un peu glissé pendant l'examen de l'accroc.)

L'emploi du possessif se laisse expliquer aisément: «The part acquires a personality of its own once it is characterized» (Hatcher, 1944, p. 459).

Le recours au possessif n'est cependant pas requis en allemand comme il l'est en français'. Ainsi l'on peut dire:

7. Ce qui ne veut pas dire que l'emploi de l'article soit impossible en français. Il est possible, mais seulement si ce que désigne l'apport est une qualité coutumière, déliée de la condition de l'instant: «Er merkte, ... wie der sonst frei und aufrecht getragene Hals zwischen die Schultern niedersank.» (Su 74) 
(10) Er hatte ... den faulen, satten Körper der Länge nach auf der Bank ausgestreckt. (Sü 58)

(Littéralement: le corps repu et paresseux)

(Il avait étendu de tout son long ... son corps repu et paresseux.)

On voit que l'allemand n'est pas tenu, en présence d'un apport lexical de type descriptif, de renoncer, comme le français, à l'article. L'article est habile, en français, à évoquer uniquement le rapport permanent qui lie la partie du corps à l'ensemble du corps et il semble, par là, incompatible avec un adjectif exprimant une qualité passagère comme satt (repu).

4. Avec les noms désignant des possessions personnelles comme casquette, valise, parapluie, - réalités qui se distinguent des parties du corps par leur caractère extrinsèque à la personne -, le traitement régulier en français est l'emploi du possessif; l'allemand, lui, emploie fréquemment l'article. Par exemple:

(11) [Er] hatte ... die Schuhe ausgezogen. (Sü 5)

(Littéralement: Il avait ôté les chaussures.)

(Il avait ôté ses chaussures.)

5. Avec le nom d'une partie du corps, lorsque celle-ci est l'objet d'un verbe qui n'est pas un verbe de mouvement, le français et l'allemand connaissent deux traitements. L'emploi du possessif:

(12) Dabei konnte sie so intime Kenntnisse seines Lebenswandels gewinnen, wie: ... ob er seine Haare gewaschen hatte. (Sü 33) (À cette occasion, elle était en mesure d'apprendre sur son compte les choses les plus intimes: ... s'il avait lavé ses cheveux.)

(Il constata que le cou, d'habitude dégagé et blen droit, rentrait dans les épaules.)

ou, comme le note G. Guillaume (1919, p. 214), si l'apport renvoie à une position comme gauche, droit, premier:

«Um die rechte Hand frei zu haben ...» (Sü 26)

(Pour avoir la main droite libre ...) 
ou l'emploi de l'article avec expression séparée de la personne sous la forme d'un pronom personnel:

(13) Er wäscht sich die Haare. (Il se lave les cheveux.)

Mais l'allemand dispose d'une possibilité inconnue du français: l'emploi de l'article seul. Comparons:

(14) Er putzte die Zähne. (Sü 25)

(Littéralement: Il brossa les dents.)

(Il se brossa les dents.)

avec:

(15) Das Mädchen putzt sich die Zähne. (Grimm, 1987, p. 24) (La jeune fille se brosse les dents.)

L'allemand peut donc dans ce type de contexte employer l'article seul, sans pronom. Cette possibilité est interdite en français.

6. Lorsque la partie du corps est sujet du verbe, l'emploi du possessif est fréquent:

(16) Sein Herz pochte. (Sü 49)

(Son cœur battait.)

Mais on rencontre souvent le traitement qui consiste à exprimer séparément la personne sous la forme d'un pronom ou d'un substantif:

(17) Das Blut stieg ihm (dem Mann) zu Kopf. (Sü 39)

(Le sang lui montait à la tête.)

Ces deux types de tournures sont analogues à celles du français comme en font foi les traductions de (16) et (17). L'allemand, cependant, connaît ici aussi une possibilité à laquelle le français est réfractaire: l'article sans expression séparée de la personne.

(18) Die Stirn war feucht. (Sü 46)

(Littéralement: Le front était humide.) 
(Son front était humide.)

(19) Die Füße brannten. (Sü 88)

(Littéralement: Les pieds brûlaient.)

(Les pieds lui brûlaient.)

(20) Die Beine schmerzten. (Sü 88)

(Littéralement: Les jambes étaient endolories.)

(Il avait les jambes endolories.)

Les traducteurs ont coutume a) de traduire l'article par le possessif comme en (18), b) d'ajouter un pronom comme en (19) ou, finalement, de recourir à une fonction autre que celle de sujet, surtout à la construction: avoir + article défini + partie du corps + attribut de l'objet (adjectif ou participe) comme en (20), construction n'ayant pas d'équivalent en allemand.

Comme on le voit à ces quelques exemples, l'allemand connaît dans la distribution des déterminants des divergences avec le français. On retiendra que l'article défini est plus utilisé en allemand qu'en français ${ }^{8}$.

4. La répartition de l'article et du possessif avec les noms de parenté

Comment s'opère en général dans les textes la répartition entre les deux déterminants avec les noms de parenté? Dans un récit où l'auteur se contente de raconter des événements ponctuels les uns à la suite des autres, la tendance du français sera d'employer le possessif exclusivement. L'allemand, lui, pourra aussi avoir recours à l'article comme on peut le voir dans l'exemple (21):

(21) Er war also vom Angeln nach Hause gekommen und in die Küche gelaufen, in der Erwartung, die Mutter dort beim Kochen anzutreffen, und da war die Mutter nicht mehr vorhanden ... und ein paar Tage später war dann auch der

8. La place nous manque ici pour nous apesantir sur les différences qui séparent des exemples comme (12) et (13). 
Vater verschwunden, und Jonathan und seine kleinere Schwester befanden sich plötzlich in einem Zug, der nach Süden fuhr. (Sü 6)

(Il revenait donc de pêcher à la ligne et avait couru à la cuisine, pensant trouver sa mère en train de préparer le repas, et voilà que sa mère n'y était plus... Quelques jours plus tard, son père avait disparu lui aussi, et Jonathan et sa petite sœur se retrouvèrent dans un train qui roulait vers le sud.)

Le traducteur s'est senti obligé de recourir au possessif pour rester fidèle à l'esprit du français. Dans ce passage, on ne saurait dire: ${ }^{*} I l$ pensa trouver la mère, mais voild que la mère n'y était plus. L'allemand, donc, qui peut employer ici les deux déterminants, peut les opposer, mais pas le français. Dans cet extrait de Die Taube, il est probable que l'article accompagne la désignation du père et de la mère parce que toute personne possède des parents, tandis que le possessif avec Schwester souligne le fait contingent de posséder une sœur.

Ce n'est pas que le français ne connaisse pas, lui aussi, l'emploi de l'article défini dans un récit, mais la simple relation d'événements ne le permet pas. Il faut en plus que les événements fassent l'objet d'une sommation. Par exemple, si l'on cite les événements importants que subsume l'existence d'une personne ou si l'on résume, comme dans l'exemple suivant, les faits saillants qui ont marqué la vie d'une famille durant quarante ans (dans un article du Zeit soulignant le $40^{\circ}$ anniversaire de la RDA), il est possible de dire, en français et en allemand:

(22) Günther K. ist Arbeiter, Schlosser ... 1961 dann der Mauerbau ... 1964 sind sie in ihr Häuschen gezogen, das gehörte einem, der in den Westen gegangen war. Seine Frau nahm eine Halbtagstelle in der Kaufhalle an. Zur Jugendweihe der Tochter 1967 gab es Karnickel aus dem eigenen Stall. Die Tochter fing an zu lernen, zwei Jahre später der Sohn. Die Kinder heirateten, inzwischen sind vier Enkelkinder da. (Die Zeit, 20 oct. 1989, p. 99)

(Gunther K. est ouvrier, serrurier ... Puis, en 1961, il y eut la construction du mur. En 1964, ils emménagèrent dans leur petite maison qui appartenaient à quelqu'un qui avait fui à 
l'ouest. Sa femme se fit employer à mi-temps dans un supermarché. En 1967, à l'occasion de la «Jugendweihe» de la fille, l'on servit du lapin élevé dans le clapier familial. La fille entra en apprentissage, suivie deux ans plus tard par le fils. Les enfants se marièrent et depuis la famille compte quatre petits-enfants.)

Ici, également, l'article (die Tochter) s'oppose au possessif (seine Frau). Toutefois, ce n'est pas le degré de parenté en soi qui est en cause ici, mais l'activité dans laquelle s'inscrit chaque personne: la désignation des personnes qui ont un comportement socialement attendu, selon une séquence immuable ( Jugendweihe», apprentissage, mariage...) se voient précédées de l'article; la désignation de la personne qui a un comportement qui n'est pas spécialement reconnu socialement, mais propre uniquement à elle (emploi à mi-temps) se voit précédée du possessif? L'opposition des deux déterminants recouvre ici celle d'un comportement institutionnalisé et d'un comportement qui ne l'est pas.

\section{Les noms de parenté dans la Métamorphose (Vater, Mutter,} Schwester, Frau, Tochter)

Si l'on examine l'emploi des noms de parenté eux-mêmes, il se dessine un plan remarquable: presque toute la nouvelle nous place du point de vue de Gregor et, c'est à partir de lui que s'organise la cellule familiale; les seules désignations sont alors Gregor, Vater, Mutter et Schwester ${ }^{10}$. Mais dès que Gregor a disparu, le point de vue change radicalement et la famille s'organise autour du père; on a alors les désignations suivantes: Herr Samsa, Frau, Tochter.

Si l'on examine à présent les déterminants qui accompagnent ces noms, les faits sont plus remarquables encore: la désignation de la

9. G. Kleiber a montré à propos du démonstratif que le choix du déterminant dépend de la façon dont est envisagée la réalité dont on parle (une personne, par exemple) et/ou l'activité dans laquelle cette personne est engagée dans le récit.

10. Seules exceptions des Sohnes (p.26), lorsque Gregor se place du point de vue de son patron vis-à-vis de la famille Samsa; et mein Bruder, dans le discours de Grete (p.166). 
mère, celle du père et celle de la sœur, se font à peu près exclusivement avec l'article (cf. infra, p. 14). Dans les quelques pages où ils apparaissent, les mots Frau et Tochter sont précédés du possessif, à l'exception de l'ultime mention de Tochter qui clôt la nouvelle; Tochter est alors précédé de l'article.

En d'autres termes, à partir du premier moment de l'épreuve de Gregor jusqu'à sa disparition, le reste de la famille forme grammaticalement un ensemble, sans que le lien qui les unit à Gregor ne soit rendu explicite, sinon par le sens lexical des substantifs en cause. L'effet résultant est une distanciation qu'ont bien vu les traducteurs du Livre de Poche: «Il y a un jeu constant dans l'usage des adjectifs possessifs, comme pour suggérer que les rapports les plus habituels (au corps, aux objets et à la famille) se brouillent dans la conscience de Gregor» (p. 15). Tout au long de la nouvelle, Gregor va se détacher, voire s'opposer aux trois autres, qui forment, face à lui, une unité.

Nous avons aussi souligné que l'emploi de l'article est possible lorsque l'ensemble que forment les parties est doté d'une certaine permanence, soit parce qu'il est toujours formé des mêmes parties, ou soit qu'il subsiste pendant une longue période de temps. Ainsi, ce que va souligner l'emploi de l'article, c'est la pérennité de la famille, ou, à tout le moins, sa persistance à travers des événements imprévisibles, chaotiques et incontrôlables auxquels elle est mêlée.

Une fois que les terribles événements sont chose du passé, l'auteur change de point de vue et il voit les événements à partir du père. Cette fois-ci, le déterminant est le possessif. Chaque individu est vu à la fois en relation au père et comme personnage autonome. À aucun moment on ne les présente comme formant un ensemble. Les événements auxquels ils participent maintenant n'ont, en effet, rien de menaçant et n'appellent aucune réaction collective, chaque personne y participant individuellement de plein gré.

La nouvelle se termine par l'évocation de die Tochter; l'article, qui suggère l'absence de lien avec le père (et avec la mère) souligne ainsi le fait que Grete aura désormais son destin propre, ce qui est bien la perspective sur laquelle s'achève le récit. 
Qu'en est-il, dans le détail, de l'emploi des deux déterminants avec les noms de parenté? Vater est employé 96 fois avec l'article et une seule fois avec le possessif, ${ }^{11}$ Mutter, 67 fois avec l'article et 1 fois avec le possessif, et Schwester, 98 fois avec l'article et 3 fois avec le possessif.

On est en droit de se demander alors à quelle intention correspond l'emploi de l'article dans une proportion qui dépasse largement la moyenne des cas et pourquoi l'auteur a eu recours quand même à quelques reprises au possessif.

1. La seule occurrence de Vater avec le possessif se trouve dans une incise à la page 36 :

$$
\begin{aligned}
& \text { Zwei starke Leute - er dachte an seinen Vater und das } \\
& \text { Dienstmaddchen - hătten vollstăndig genügt. (Ka } 1 / 36 \text { ) } \\
& \text { (Deux personnes vigoureuses - il songea à son père et à la } \\
& \text { bonne - auraient largement suffi. [Ka } 1 / 37] \text { ) }
\end{aligned}
$$

Il est fort probable qu'il y a ici un principe de différenciation en jeu: le père et la bonne ne se situent pas dans la même sphère; la seconde est étrangère à la famille et la désignation de celle-ci ne peut survenir qu'avec l'article comme partie d'un autre ensemble que sont les gens de maison (die Köchin, die Bedienerin .../ la cuisinière, la servante). La seule façon qu'il y avait d'éviter que le père et la bonne ne puissent être perçus comme faisant partie d'un ensemble commun, ce qu'aurait suggéré le double emploi de l'article, a été pour Kafka d'utiliser le possessif avec celui des deux mots qui l'autorisait.

2. Le seul emploi du possessif avec Mutter se trouve à la page 66:

(24) Als er da schaukelnd vor verhaltener Bewegung, gar nicht weit von seiner Mutter entfernt, ihr gerade gegenüber auf dem Boden lag, sprang diese ... in die Hőhe. (Ka 1/66)

11. Nous n'avons pas tenu compte des groupes de deux substantifs coordonnés sans article (Vater und Mutter) puisque cette construction autorise l'article zéro. De même, il n'a pas été tenu compte de l'apostrophe dans le discours direct «Vater!» 
(S'étant approché tout près de sa mère et se balançant encore dans son élan, il se trouva sur le plancher juste en face d'elle,... elle sauta tout à coup en l'air. [Ka 1/67])

Quel est le contexte? Gregor plaide sa cause devant le gérant, qui prend la fuite; le malheureux Gregor cherche à expliquer qu'il a sa famille à sa charge et, à ce moment, il se trouve tout près de sa mère qui prend soudain peur à sa vue. Devant la réaction de sa mère, Gregor lève les yeux vers elle et l'interpelle. C'est l'unique moment du récit où il marque son intention de lui parler directement (et non simplement en réponse à une question comme à la page 28). L'effet résultant est le même que celui dont nous allons parler maintenant.

3. Le nom de la sœur apparait à trois reprises avec le possessif. Une première fois en conjonction avec Eltern au début du récit. Voilà un exemple où l'on peut très bien voir la distance qui sépare les deux déterminants. En effet, Kafka écrit d'abord:

(25) [Gregor] fühlte ... einen großen Stolz darüber, daß er seinen Eltern und seiner Schwester ein solches Leben in einer so schönen Wohnung hatte verschaffen können. (Ka 1/76)

(Gregor éprouva une grande fierté d'avoir pu permettre à ses parents et à sa scur d'avoir une vie pareille, dans un si bel appartement. [Ka 1/77])

puis, un paragraphe plus loin:

(26) Spät erst in der Nacht wurde das Licht im Wohnzimmer ausgeloscht, und nun war leicht festzustellen, daß die Eltern und die Schwester so lange wachgeblieben waren, denn wie man genau hören konnte, entfernten sich jetzt alle drei auf den Fußspitzen. (Ka 1/78)

(Ce fut seulement tard dans la nuit que la lumière s'éteignit dans la salle, et il fut aisé de constater que les parents et la scur avaient veillé jusque-là, car comme on put parfaitement l'entendre, ils s'éloignèrent tous trois sur la pointe des pieds. [Ka 1/79]) 
Dans le premier cas, Kafka évoque les sentiments qu'éprouve Gregor à l'endroit de ses proches. Dans le second, il ne s'agit plus que d'un événement dont Gregor est témoin et auquel il ne participe pas. Les deux autres exemples de possessif avec Schwester se trouvent à l'endroit du récit où il est raconté que Gregor, jugeant que les locataires ne prêtent pas une oreille assez attentive à sa sœur qui joue du violon, se précipite vers elle pour la convaincre de rester près de lui, seul capable de la comprendre. On sait le résultat malheureux de son geste. Dans les deux exemples suivants, c'est le lien privilégié que Gregor sent exister entre lui et sa sœur qui a amené le possessif à cet endroit du récit:

(27) Aber Gregor fiel es doch gar nicht ein, irgend jemandem und gar seiner Schwester Angst machen zu wollen. (Ka 1/170)

(Cependant Gregor ne songeait pas du tout à effrayer qui que ce soit, et surtout pas sa sceur. [Ka 1/171])

(28) Seine Meinung darüber, daß er verschwinden müsse, war womöglich entschiedener als die seiner Schwester. (Ka 1/174) (Son opinion quant au fait qu'il devait disparaître était peutêtre encore plus catégorique que celle de sa saur. [Ka 1/175])

On voit que ce que marque le possessif avec Schwester et Mutter, c'est le lien qui unit Gregor aux deux personnes, sa sœur et sa mère, dans les rares situations où il recherche un contact direct avec elles ou lorsqu'il est question des sentiments qu'il éprouve à leur égard.

L'emploi rare que fait Kafka du possessif a l'avantage de lui permettre de privilégier certaines situations où il peut évoquer un rapprochement entre les personnages, alors que, la plupart du temps, l'article suggère leur irréversible éloignement. Est-il possible de rendre, en traduction, ce qu'exprime Kafka lorsqu'il fait usage de l'article? C'est ce qu'il y a lieu maintenant de voir. 


\section{Les traductions des noms de parenté dans la Métamorphose $e^{12}$}

Comment les déterminants chez Kafka ont-ils été rendus dans les quatre traductions de la Métamorphose ${ }^{13}$ ? On observe que Vergne-Cain et Rudent sont restés près du texte en ce qui a trait aux déterminants, afin de conserver l'esprit du texte (cf. Préface). Vialatte fait de même, mais sans donner d'explication quant à son choix; chez lui, cette fidélité au texte est un peu paradoxale, étant donné que c'est celui des quatre traducteurs qui prend le plus de liberté vis-à-vis du vocabulaire et de la syntaxe.

Les deux autres traducteurs ont choisi de traduire l'article défini de l'original tantôt par l'article, tantôt par le possessif. Il est intéressant de voir comment cette répartition a été faite. Nous n'avons examiné que la traduction de B. Lortholary et uniquement les emplois avec le substantif Vater.

1. Considérons d'abord la traduction de der Vater, en fonction sujet ( 52 occurrences). Il apparaît que le traducteur a opéré une répartition des déterminants selon plusieurs principes:

a) Il a gardé l'article défini (der Vater/le père), lorsque le syntagme est sujet d'un verbe signifiant «dire» accompagné des paroles mêmes prononcées par le locuteur (discours direct) (11 occurrences). Par exemple:

(29) «Sie hat tausendmal recht, sagte der Vater für sich.» (Ka 1/166)

(Elle a mille fois raison, dit le père à part lui. [Ka 2/87])

12. A propos de l'ensemble des traductions, nous ne pouvons avancer de chiffres absolus parce qu'il n'y a pas que les deux déterminants qui soient en cause (die Mutter et seine Mutter). Beaucoup d'occurrences ont été traduites 1) par un nom propre (Grete);2) par des pronoms personnels (il et elle); 3) par un complément déterminatif (la mère de Gregor).

13. Nous ne considérons que la traduction de l'article défini dans le texte de Kafka. Chaque fois que ce demier a utilisé le possessif, les traducteurs ont gardé le même déterminant. 
Son interlocuteur est, à deux exceptions près, quelqu'un d'autre que Gregor. La seule fois où Gregor est son interlocuteur et que der Vater est traduit par le pere, celui-ci se montre aggressif envers lui:
«Also kann der Herr Prokurist schon zu dir hinein? fragte der ungeduldige Vater.» (Ka 1/44)
(«Alors, est-ce que M. le fondé de pouvoir peut venir te voir, maintenant? demanda impatiemment le père.» [Ka 2/33])

La seule fois où der Vater est traduit par son père, c'est lorsque le père s'adresse à Gregor sur un ton de sollicitude:

«Gregor, sagte nun der Vater aus dem Nebenzimmer links, der Prokurist ist gekommen.» (Ka 1/40)

( «Gregor, dit alors son père dans la chambre de gauche, M. le fondé de pouvoir est là». [Ka 2/32])

Le fait s'explique aisément puisqu'il y a, d'une part, une homologie entre le père, la mère et la scur, parties du tout qu'est la famille, et le locuteur et l'allocutaire, parties du tout qu'est la situation d'interlozution. D'autre part, le fait que l'article n'exprime pas un lien entre les personnes et Gregor fait que son emploi est le plus approprié chaque fois que Gregor n'est pas directement en cause ou qu'il est rejeté.

b) On observe aussi la tendance du traducteur à conserver l'article, lorsque le comportement dans lequel est impliqué le père n'est pas un événement ponctuel, mais répété sur une longue période de temps (8 occurrences). Par exemple:

(32) Der Vater schlief bald nach dem Nachtessen in seinem Sessel ein... Manchmal wachte der Vater auf, und als wisse er gar nicht, daß er geschlafen habe, sagte er zur Mutter ... und schlief sofort wieder ein, während Mutter und Schwester einander müde zulächelten. (Ka 1/134)

(Le père s'endormait sur sa chaise un peu après la fin du dîner... Parfois le père se réveillait et, ... disait à la mère ... Puis il se rendormait aussitôt, tandis que la mère et la sœur échangeaient des sourires. [ $\mathrm{Ka} 2 / 74]$ ) 
Par contre, si l'événement est répété dans un laps de temps très court ( 2 occurrences), c'est le possessif que le traducteur emploie:

Und so lief er vor dem Vater her, stockte, wenn der Vater stehen blieb, und eilte schon wieder vorwärts, wenn sich der Vater nur rührte. (Ka 1/128)

(Aussi se mit-il à courir devant son père, s'arrêtant quand son père s'immobilisait et filant à nouveau, dès que son père faisait un mouvement. [Ka 2/69])

ou si l'habitude en question est une habitude qui est reliée à la vie de Gregor depuis sa transformation ( 2 occurrences). Par exemple:

(34) Im Wohnzimmer war, wie Gregor durch die Türspalte sah, das Gas angezündet, aber wăhrend sonst zu dieser Tageszeit der Vater seine nachmittags erscheinende Zeitung der Mutter und manchmal auch der Schwester mit erhobener Stimme vorzulesen pflegte, hörte man jetzt keinen Laut. (Ka 1/76)

(Dans la salle de séjour, Gregor vit par la fente de la porte que l'éclairage au gaz était allumé, mais alors que d'habitude c'était l'heure où son père lisait d'une voix forte à sa mère et parfois aussi à sa sceur, le journal paraissant l'après-midi, on n'entendait cette fois pas le moindre son. [Ka 2/48])

Ici encore, il y a homologie entre un comportement qui s'étend dans le temps, qui représente une habitude par sa répétition et le fait que le désigné est vu comme faisant partie d'un ensemble dont la définition exige qu'il ne soit pas fortuit, mais qu'il ait une certaine permanence dans la durée.

Si l'événement est ponctuel (16 occurrences), le traducteur a recours au possessif:

(35) Der Vater kehrte auch zu seinem Frühstück zurück. (Ka 1/30) (Son père retourna à son petit déjeuner. [Ka 2/27])

Il reste à expliquer les cas où le traducteur maintient l'emploi de l'article, même lorsque l'événement est unique, c'est-à-dire non appelé à se répéter (12 occurrences). Les raisons qu'il y a d'employer 
l'article sont diverses. On note que le traducteur rend der Vater par le pere, si ce dernier est dans une situation où sa présence n'est pas recherchée, par exemple lorsque la mère et la sœur profitent de son absence pour ranger la chambre de Gregor. L'article est maintenu également chaque fois que le personnage du père s'oppose comme membre de la famille à tout ce qui est extérieur à elle comme, par exemple, le groupe des trois locataires. On a aussi l'article chaque fois que le père est dans une situation où il accomplit avec sa femme et sa fille, une tâche commune au cours de laquelle chacun remplit un rôle. Prenons:

(36) Bald kam der Vater mit dem Notenpult, die Mutter mit den Noten und die Schwester mit der Violine. (Ka 1/154)

(On vit bientôt arriver le père avec le pupitre, la mère avec la partition et la sœur avec son violon. [Ka 2/83])

L'article est habile à suggérer que chaque individu a un rôle spécifique dans une tâche commune.

2. Lorsque le substantif est à l'accusatif, sans préposition (12 occurrences) ${ }^{14}$, on s'aperçoit que le traducteur a choisi l'article lorsque le sujet de la phrase n'est pas Gregor (7 occurrences):

(37) Die Mutter ... sah zuerst mit gefalteten Händen den Vater an. (Ka 1/56)

(La mère commença par regarder le père en joignant les mains. [Ka 2/39])

et le possessif lorsque Gregor est sujet de la phrase (3 occurrences):

14. Il est important d'exclure les cas où le possessif se rapporte au sujet quand celui-ci n'est pas Gregor : la sémiologie du possessif ne permettant pas de reconnaître le possesseur à coup sûr, l'interprétation courante est alors de considérer la troisième personne incluse dans le possessif comme représentant le sujet et non Gregor (2 occurrences). Par exemple, son se rapportant à Grete: «Den Vater wagte sie nicht, um Hilfe zu bitten.» (Ka 1/108) (Son père, elle n'osait pas lui demander de l'aider. [Ka 2/61]) 

hung ungeduldig zu machen. (Ka $1 / 70$ )

(Il craignait d'impatienter son père en perdant du temps à se retourner. [Ka 2/44])

La polarisation est nette.

3. Le principe vaut également dans le cas des emplois prépositionnels (13 occurrences) ${ }^{15}$. On a un seul exemple où le traducteur a recours à l'article, et son analyse s'avère délicate:

[Er] sah ... noch, wie vor der schreienden Schwester die Mutter hervoreilte, im Hemd, denn die Schwester hatte sie entkleidet, um ihr in der Ohnmacht Atemfreiheit zu verschaffen, wie dann die Mutter auf den Vater zulief und ihr auf dem Wege die aufgebundenen Röcke einer nach dem anderen zu Boden glitten und wie sie stolpernd über die Röcke auf den Vater eindrang. (Ka 1/130)

(Il vit seulement que..., suivie par sa sœur qui criait, sa mère ... sortait précipitamment [de sa chambre], en chemise, car sa sœur l'avait déshabillée pour qu'elle respirât plus librement pendant son évanouissement, en perdant en chemin, l'un après l'autre, ses jupons délacés qui glissaient à terre, puis que sa mère courait vers son père, et qu'en trébuchant sur eux elle se précipitait sur le père. [Ka 2/70])

Il est probable que le traducteur a été porté à organiser les éléments par paires avec le même déterminant: sa mère courait vers son pere; il lui aura semblé possible dans la seconde phrase de dire le pere, profitant

15. La traduction d'un complément prépositionnel donne lieu très souvent à des transpositions : le syntagme remplit une tout autre fonction dans le texte d'arrivée; c'est pourquoi il devient difficile de les comparer. Nous n'avons analysé que les compléments prépositionnels trouvant un équivalent exact dans la traduction. Pour la même raison, nous ne traiterons pas des 4 occurrences de Vater au datif ou de ses 12 occurrences au génitif. En effet, ces derniers sont rarement traduits par des compléments d'attribution ou des compléments du nom. 
de ce que le sujet est un pronom et non un syntagme avec un déterminant: elle se précipitait sur le père.

On voit avec l'exemple de Vater que le choix du déterminant est toujours dicté par l'affinité que le traducteur perçoit entre la situation des personnes dans le récit et l'impression que livre chacun des déterminants. Ainsi, un comportement répété est senti en affinité avec l'impression de quelque chose qui fait partie à titre permanent d'un ensemble, impression liée à l'article défini. Un comportement en rapport direct avec Gregor sera senti en affinité avec le possessif par lequel, précisément, il est établi un rapport à Gregor par le truchement de la troisième personne qui y est incluse.

On aura noté une grande convergence dans certains cas; par exemple, der Vater, sujet d'un verbe signifiant «dire» est régulièrement traduit par le père. Mais, dans d'autres cas, la situation est sujette à interprétation. Ainsi, dans une situation où le père a un comportement hostile envers son fils, un traducteur pourra être sensible au lien étroit que supposent de tels sentiments entre deux personnes et le possessif lui semblera adéquat; un autre considérera que l'hostilité constitue un lien négatif, assimilable à une absence de lien, et l'article lui semblera plus approprié.

Ainsi les situations comme les interprétations peuvent être diverses, mais les impressions - en petit nombre - qui sont liées à chaque déterminant et que nous avons analysées dans les sections 1 . et 2. (pp. 3 et 5) sont invariantes.

\section{Conclusion}

Que peut-on retenir des quelques faits que nous avons examinés? Pour ce qui est du texte original, on voit que Kafka a exploité d'une façon particulière une possibilité que lui offre sa langue, et qui existe aussi en français: celle du choix entre l'article défini et le possessif. Kafka a choisi dans la très grande majorité des cas l'emploi de l'article défini.

Le traducteur, lui, tenu de choisir l'option qu'il estime la meilleure, doit décider si sa traduction va reproduire ou non la grammaire du texte de départ. Nous avons vu les deux choix les plus 
caractéristiques: celui de Vergne-Cain et Rudent qui ont traduit à peu près tous les articles du texte original par l'article en français, et celui de Lortholary qui a préféré employer dans sa traduction les deux déterminants.

Nous avons vu qu'il n'y a pas, en l'affaire, de principe contraignant. Cependant, lorsque tous les traducteurs, à l'exception de ceux de l'édition du Livre de Poche, emploient le possessif dans des exemples comme:

(40) Gregor glaubte sogar einen dankbaren Blick erhascht zu haben, als er einmal mit dem Kopf vorsichtig das Leintuch ein wenig lüftete, um nachzusehen, wie die Schwester die neue Einrichtung aufnahm. (Ka 1/104)

(Gregor crut même avoir saisi un regard reconnaissant, une fois qu'il soulevait prudemment le tissu avec la tête pour regarder comment la sœur réagissait à ce nouveau dispositif.) [Ka 1/105]

(... comment sa sœur prenait ce changement de situation. [Ka 2/60])

(... pour voir comment sa sœur appréciait sa nouvelle organisation. [Ka 3/114])

(... pour observer l'impression que causait à sa scur le nouvel agencement. [Ka 4/49])

L'impression ne laisse pas d'être significative. Il faut alors conclure que dans une langue comme l'allemand qui connaît un usage plus large de l'article défini (dans les contextes où il peut alterner avec le possessif), celui-ci est moins marqué stylistiquement qu'il ne l'est en français, langue qui fait une plus large place au possessif. En d'autres termes, l'emploi de l'article est moins «exceptionnel» en allemand qu'il ne l'est en français. C'est pourquoi être fidele à la grammaire du texte original ne signifie pas nécessairement que l'on fait justice au texte que l'on a à traduire. C'est ce que nous avons tenté de montrer même si notre connaissance des systèmes de l'article et du possessif dans chacune des deux langues est encore incomplète.

Université Laval 


\section{Références}

GRIMM, Brüder (1958). Buntes Spiel (Märchen). Insel-Bücherei 443, Insel-Verlag. [Gr]

HANDKE, Peter (1974). Die Angst des Tormanns beim Elfmeter. Suhrkamp Taschenbuch 27, Frankfurt/M., Suhrkamp Verlag, 112 p. [Ha]

KAFKA, Franz (1988). Die Verwandlung / La Métamorphose. Paris, Le Livre de poche, Les Langues modernes / bilingue, traduction: Brigitte Vergne-Cain et Gérard Rudent. [Ka 1]

KAFKA, Franz (1988). Die Verwandlung / La Métamorphose. Paris, Flammarion, coll. «GF», 510, traduction: Bernard Lortholary. [Ka 2]

KAFKA, Franz (1989). Die Verwandlung / La Métamorphose. Paris, Gallimard, coll. «Folio», 2017, traduction: Claude David. [Ka 3]

KAFKA, Franz (1955). Die Verwandlung / La Métamorphose. Paris, Gallimard, coll. «Folio», 74, traduction: Alexandre Vialatte. [Ka 4]

KOEPPEN, Wolfgang (1974). Tauben im Gras. Frankfurt-am-Main, Suhrkamp Verlag, Bibliothek Suhrkamp 393. [Koe]

SEGHERS, Anna (1970). Das siebte Kreuz. Rowohlt Taschenbuch Verlag, Reinbek bei Hamburg, RoRoRo Band 751-752. [Segh]

SÜSKIND, Patrick (1987). Die Taube. Zürich, Diogenes Verlag. [Sü]

FRENZEL, H.A. / FRENZEL, E (1969). Daten deutscher Dichtung. Band II, München, DTV.

GRIMM, Hans Jürgen (1987). Lexikon zum Artikelgebrauch. Leipzig, Verlag Enzyklopădie. 
GUILLAUME, Gustave (1919). Le Probleme de l'article et sa solution dans la langue française. Paris-Québec, A.-G. Nizet / Presses de l'Université Laval, 1975, 318 p.

HATCHER, Anna Granville (1944). «Il tend les mains/Il tend ses mains». Studies in Philology, 41, pp. 457-481.

KLEIBER, Georges (1986). «Pour une explication du paradoxe de la reprise immédiate». Langue française, 72, pp. 54-79. 